\title{
Effects of Orbital Motion on the Velocity Field of Boundary Layer Flow over a Rotating Disk
}

\author{
Mizue Munekata, Takaomi Utatsu, Hiroyuki Yoshikawa, Yasuhiro Okumura \\ Department of Mechanical System Engineering, Kumamoto University, Kumamoto, Japan \\ Email: munekata@kumamoto-u.ac.jp
}

How to cite this paper: Munekata, M., Utatsu, T., Yoshikawa, H. and Okumura, Y. (2017) Effects of Orbital Motion on the Velocity Field of Boundary Layer Flow over a Rotating Disk. Open Journal of Fluid Dynamics, 7, 169-177.

https://doi.org/10.4236/ojfd.2017.72011

Received: March 28, 2017

Accepted: May 15, 2017

Published: May 18, 2017

Copyright $\odot 2017$ by authors and Scientific Research Publishing Inc. This work is licensed under the Creative Commons Attribution International License (CC BY 4.0).

http://creativecommons.org/licenses/by/4.0/

\begin{abstract}
The purpose of this study is to investigate experimentally the effects of orbital motion on the velocity field of boundary layer flow over a rotating disk. The characteristics of velocity field at a fixed orbital angular section measured by a hot-wire anemometer show that the structure of the 3-dimensional boundary layer flow is deformed elliptically and displaced in a certain direction that is not in the orbital radial direction, but the direction of deformation depends on the combination of orbital and rotational directions. For coincide orbital and rotational directions, there are regions where the intensity of low-frequency disturbances increases rapidly in a certain central region (laminar region under pure rotation). The transient vortices, which form streaks on the coating film, are considered to be destroyed by low-frequency disturbances. However, for opposite orbital and rotational directions, the low-frequency disturbances are not observed in any section. As the adding orbital speed increases, the intensity of velocity fluctuations in the turbulence region becomes larger in the expected except in a certain region. This location of the region also depends on the direction of deformation or the combination of orbital and rotational directions.
\end{abstract}

\section{Keywords}

Boundary Layer Flow, Rotating Disk, Transition, Vortices, Hot-Wire Anemometer

\section{Introduction}

Flow fields over rotating disks appear in the context of spin-coating manufacture of semiconductors. Many studies have been accomplished both theoretical [1] and experimental [2] [3] [4] [5]. If the Reynolds number of the boundary layer flow over a rotating disk, based on the local radius from the center of disk and the rotating speed, exceeds a critical value, thirty transient vortices appear at the 
outer periphery of the laminar region for the rotating disk [6]. These vortices prevent the film from forming a uniform thickness. Instead, streaks form on the coated film because these vortices remain stationary relative to the rotating disk. We have refined our original technique using orbital motion to prevent such streaking [7].

In our earlier work, we found that the laminar region narrows and the transition point moves inward on the disk, regardless of the direction of rotation as the applied orbital rotating speed increases [7]. Moreover, we examined the characteristics of the transient vortices and low-frequency disturbances by finding the power spectra of the velocity fluctuation [7] and endeavored to visualize these vortices [8]. However, the flow field on the rotating disk under orbital motion has still not been revealed, and the effects of orbital motion on the boundary layer flow over the rotating disk flow at high Reynolds number have not been investigated.

The objective of this study is to investigate this velocity field using a larger disk than in the previous study. The effects of the velocity field in the turbulence region, the deformation of boundary layer structure, and the characteristics of disturbances are presented based on measurements obtained using a hot-wire anemometer.

\section{Experimental Apparatus and Procedure}

\subsection{Experimental Apparatus and Coordinate System}

The experimental apparatus consists of a rotating disk mounted on an orbital rotation base (Figure 1). The disk is given added orbital motion by rotating the orbital base. The disk radius $R$ is $150 \mathrm{~mm}$, and the radius of the base $R_{\mathrm{o}}$ is $50 \mathrm{~mm}$ $(=R / 3)$. The counterclockwise direction is taken as positive direction for both rotation and revolution (i.e., orbital movement). The angular speeds of rotation and revolution are controlled independently. Experimental trials were performed at a rotational velocity $N$ of $+3000 \mathrm{rpm}$ and orbital speeds $N_{\mathrm{o}}$ in the range of $-500 \mathrm{rpm} \leq N_{\mathrm{o}} \leq+500 \mathrm{rpm}$ stationary.

Two distinct coordinate systems are used. In the fixed coordinate system, the origin $\mathrm{O}_{\mathrm{o}}$ is the center of the orbit, and the radial distance from $\mathrm{O}_{\mathrm{o}}$ is denoted $r_{\mathrm{o}}$. In the moving coordinate system, the origin $\mathrm{O}$ is the center of disk. The coordinates of the two systems are denoted $r, \theta$, and $z$ for the distance in the radial direction, the angle in the circumferential direction, and distance in the axial direction, respectively. As indicated in Figure 2, if the line connecting the orbit center $\mathrm{O}_{\mathrm{o}}$ and the disk center $\mathrm{O}$ is on line $\mathrm{AB}$ in the fixed coordinate system, directions $\mathrm{OA}\left(\theta=180^{\circ}\right), \mathrm{OB}\left(\theta=0^{\circ}\right), \mathrm{OC}\left(\theta=90^{\circ}\right)$, and $\mathrm{OD}\left(\theta=270^{\circ}\right)$ in the radial direction will be denoted by $r_{\mathrm{A}}, r_{\mathrm{B}}, r_{\mathrm{C}}$, and $r_{\mathrm{D}}$, respectively.

\subsection{Hot-Wire Measurement}

The velocity field of the boundary layer flow on the disk is measured with a single hot-wire anemometer at a fixed angular position using a timing-mark laser sensor. The hot-wire is positioned parallel to the disk surface and aligned normal 

(1) Disk
(2) Orbital base
(3) Pulley
(4) Timing belt
(5) Rotation motor
(6) Orbital motor
(7) Timing mark laser sensor
(8) Inverter
(9) Timer

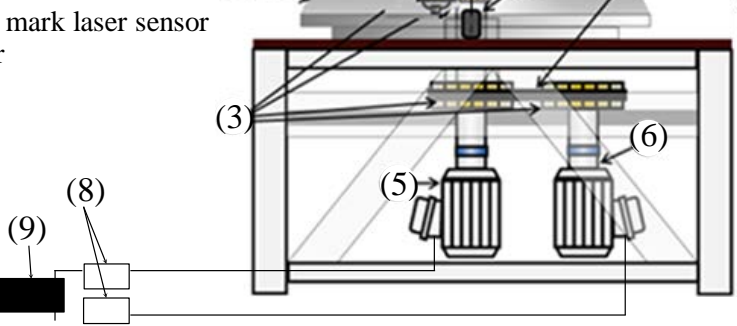

Figure 1. Experimental apparatus.

to the rotation. Hence the tangential (i.e., circumferential) velocity $v_{\theta}$ is measured. The velocity data of 250 points are sampled at all radial position on the disk, and the mean tangential velocity $V_{\theta}$ is calculated. An analog-to-digital converter is triggered by a timing pulse signal from the orbital base; this ensures that the center of each time record represents the same orbital angular position. The velocities at 1024 points are sampled at every trigger signal for at most 750 orbital revolutions at a sampling frequency of $100 \mathrm{kHz}$ (low-pass filter: $12 \mathrm{kHz}$ ).

The radial boundary layer is measured at a height $z=0.65 \mathrm{~mm}$ from the disk's surface (so that $z / \delta=3.0$ ). Here $\delta$ is the thickness of the boundary layer in the pure-rotation case defined as $\delta=(v / \omega)^{1 / 2}$ ( $v$. kinematic viscosity of air, $\omega$ : angular velocity of a rotation disk).

\section{Results and Discussion}

\subsection{Mean Velocity Profile}

Figure 3 shows the profile of the mean tangential velocity component on the disk $\left(V_{\theta}\right)$ at a given orbital angular position, where the orbit center $\mathrm{O}_{\mathrm{o}}$ is on the measurement section AOB (Figure 2). Figure 3(a) and Figure 3(b) shows instances of coincident and opposite rotations of the orbital and rotational directions, respectively. The circle in these figures represents the periphery of the disk. The other measurement section is orthogonal to the AOB section at the center axis ( $z$-axis) of the disk. The mean tangential velocity is normalized by the tangential velocity $R \omega$ at the periphery of the spinning disk. Here, $\omega$ denotes the absolute value of the angular velocity of the spinning disk. The velocities $V_{\theta}$ in the OC direction $\left(r_{\mathrm{C}}\right.$ direction) and $\mathrm{OB}$ direction $\left(r_{\mathrm{B}}\right.$ direction) are defined as positive.

Under pure rotation, the velocity data at $r / R \leq 0.55$ in any section accord with the theoretical values in laminar flow on a purely rotating disk given by von Karman [1]. The boundary layer transition from a laminar flow to a transient flow occurs at around $r / R=0.6$, and the boundary layer transition to a turbulent flow occurs at around $r / R=0.75$, as seen in the changes in the velocity gradient in the radial direction. 


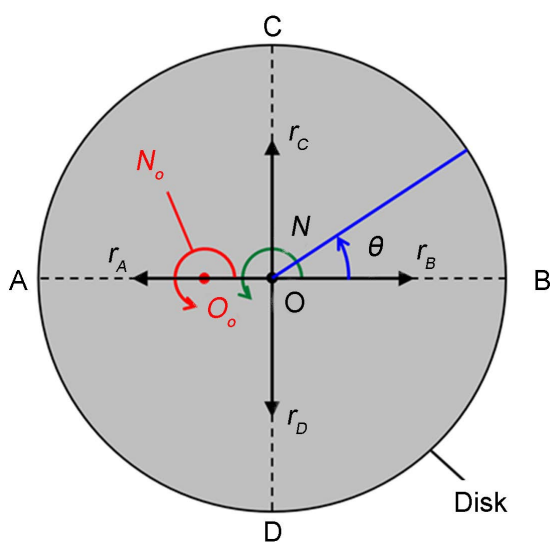

Figure 2. Measurement section.

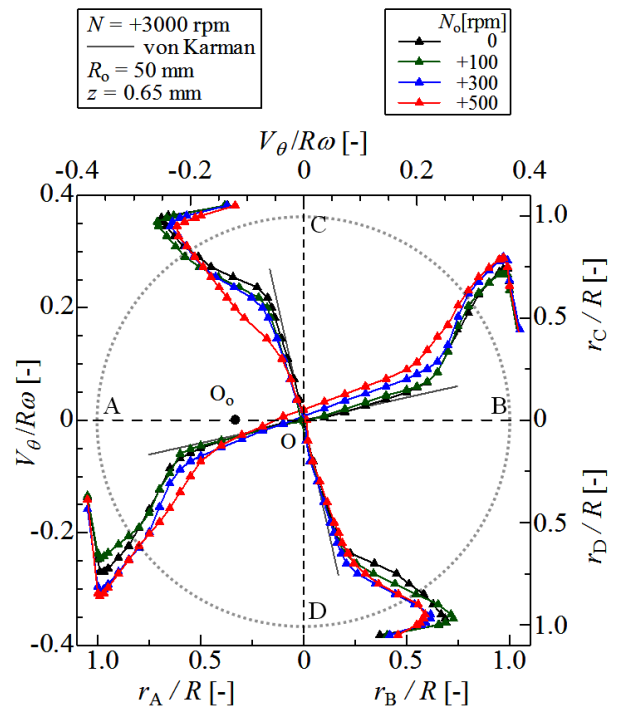

(a)

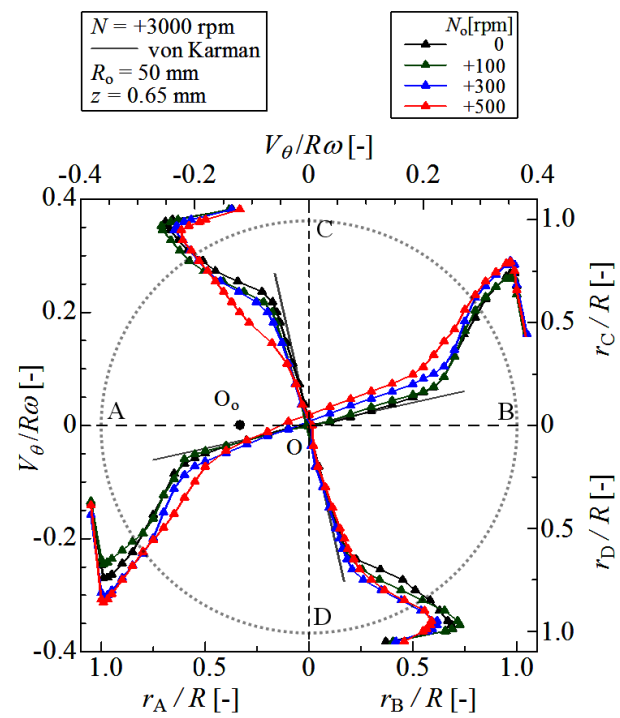

(b)

Figure 3. Radial distributions of the mean tangential velocity. (a) Coincident orbital and rotational directions; (b) Opposite orbital and rotational directions. 
In Figure 3(a), for the central region of the disk (laminar region) with linear distribution of velocity, the tangential velocity and the velocity gradient at the OB section side on $\mathrm{AOB}$ section (in the orbital radial direction) under coincident orbital and rotational directions increase significantly with orbital speed. The velocity at the OC section also increases slightly with increasing the orbital speed, however the velocity gradient in the COD section (perpendicular to the orbital radial direction) has no large effect arising from the orbital motion.

From these velocity distributions, the point of $V_{\theta}=0$ moves towards $r_{\mathrm{A}}$ and $r_{\mathrm{D}}$. It is found that the mean center of spiral flow is located in the AOD region $(r-\theta$ plane at $180^{\circ}<\theta<270^{\circ}$ ) and moves increasingly away from the origin of the disk as orbital speed increases. Additionally, both the transition points from the boundary layer flow to transient flow and then to turbulent flow, at the $\mathrm{OA}, \mathrm{OB}$, and OC sections, move radially inward with increasing $V_{\theta}$. That is, the boundary transitions occur earlier. However, at the OD section, the transition points move radially outward along $r_{\mathrm{D}}$. The structure of the 3-dimensional boundary layer flow is deformed elliptically and displaced to the D-side under orbital motion.

In Figure 3(b), the velocity gradient of the linear distribution in the OA and OD sections under opposite orbital and rotational directions has a tendency to be larger with increasing negative orbital speed. The velocity increases rapidly at the OA section, and the accelerating section is on the opposite side compared with the case of coincide rotational direction. Therefore, the center of the spiral flow on the disk is located on the opposite side (BOC region) compared with the case of coincide rotational direction.

With increasing negative orbital speed, both transitions in boundary layer flow (laminar to transient and laminar to turbulent) are promoted earlier at the $\mathrm{OB}$ and OD sections; however, they are delayed at the OA and OC sections with increasing negative orbital speed. With $N_{\mathrm{o}}=-300$ and $-500 \mathrm{rpm}$, the laminar region expands and the laminar-transient transition is delayed, although it is remarkable that the mean velocity at the OA section significantly increases between the laminar region and the turbulence region. It is considered that the velocity on the surface of the disk along $\mathrm{O}$ to $\mathrm{O}_{\mathrm{o}}$ is accelerated, and the thickness of the boundary layer along $\mathrm{O}_{\mathrm{o}}$ to $\mathrm{A}$ increases because surface velocity decreases.

\subsection{Fluctuating Velocity}

The radial distributions of the root mean squared (rms) values of the fluctuating tangential velocity at the same orbital angular position $V_{\theta, \text { rms }}$ are shown in Figure 4. For pure rotation, the intensity of fluctuation in velocity is almost zero around the center of the rotation (in the laminar region) and increases rapidly in the transition region, where the radial component of the fluctuation intensity gradient is larger than in the turbulence region.

In Figure 4(a), for coincident orbital and rotational directions, the onset of the increase in fluctuation intensity moves with the shift in the boundary layer transition point arising from the addition of orbital motion. However, focusing on the OB and $\mathrm{OC}$ sections at $N_{\mathrm{o}}=+500 \mathrm{rpm}$, the fluctuation intensity becomes 


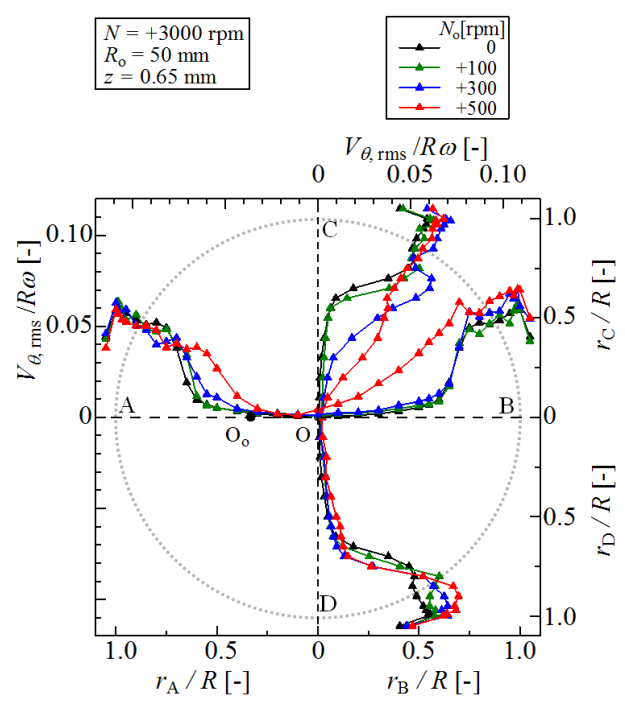

(a)

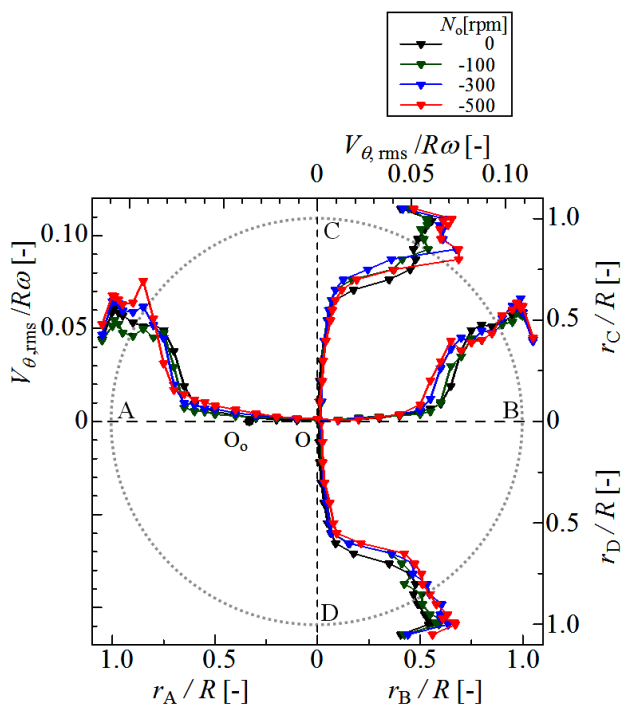

(b)

Figure 4. Radial distributions for the intensity of the tangential velocity fluctuations. (a) Coincident orbital and rotational directions; (b) Opposite orbital and rotational directions.

larger in the central region where it is considered to be laminar flow $(r / R \leq 0.5)$ from the linear velocity profile (see Figure $3(\mathrm{a})$ ). The time histories for the instantaneous tangential velocity $v_{\theta}$ at $r / R=0.3$ in this region and each section at $N_{\mathrm{o}}=-500 \mathrm{rpm}$ are given in Figure 5. Disturbances are not observed at $r_{\mathrm{A}} / R=$ 0.3 and $r_{\mathrm{D}} / R=0.3$ in the OA and OD sections. However, the velocities at $r_{\mathrm{B}} / R=$ 0.3 and $r_{\mathrm{C}} / R=0.3$ in the $\mathrm{OB}$ and $\mathrm{OC}$ sections have disturbances over long periods (low frequencies) and with large amplitudes. Consequently, it is found that the fluctuation intensity increases in this region (BOC). These low-frequency disturbances are no different from transient vortices, which cause velocity fluctuations with periods of around 12 degrees as shown at $r / R=0.65$ (in the transition region) in the pure-rotation case in Figure 6. 


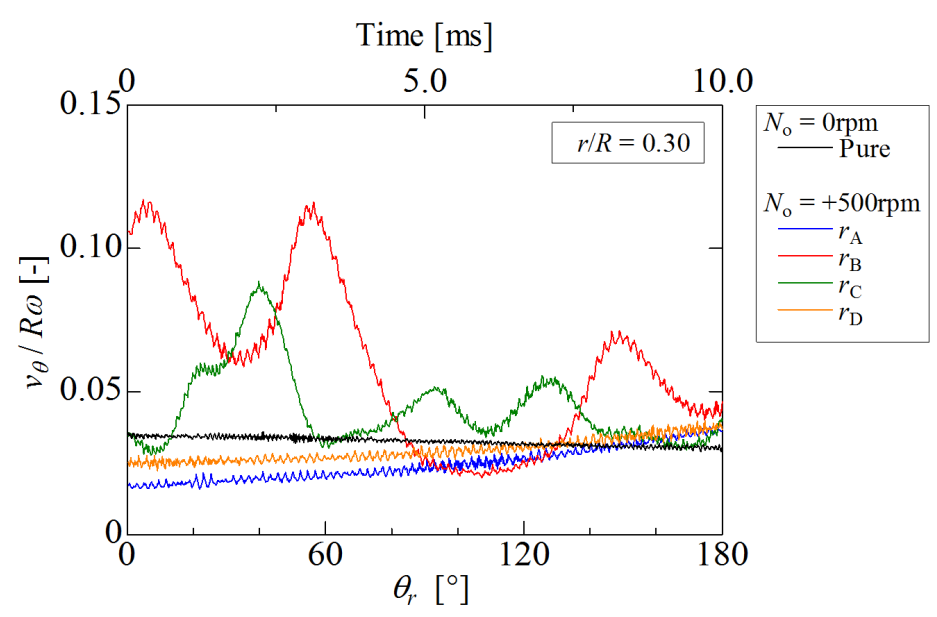

Figure 5. Time histories of $v_{\theta}$ at $r / R=0.30(N=+3000 \mathrm{rpm})$.

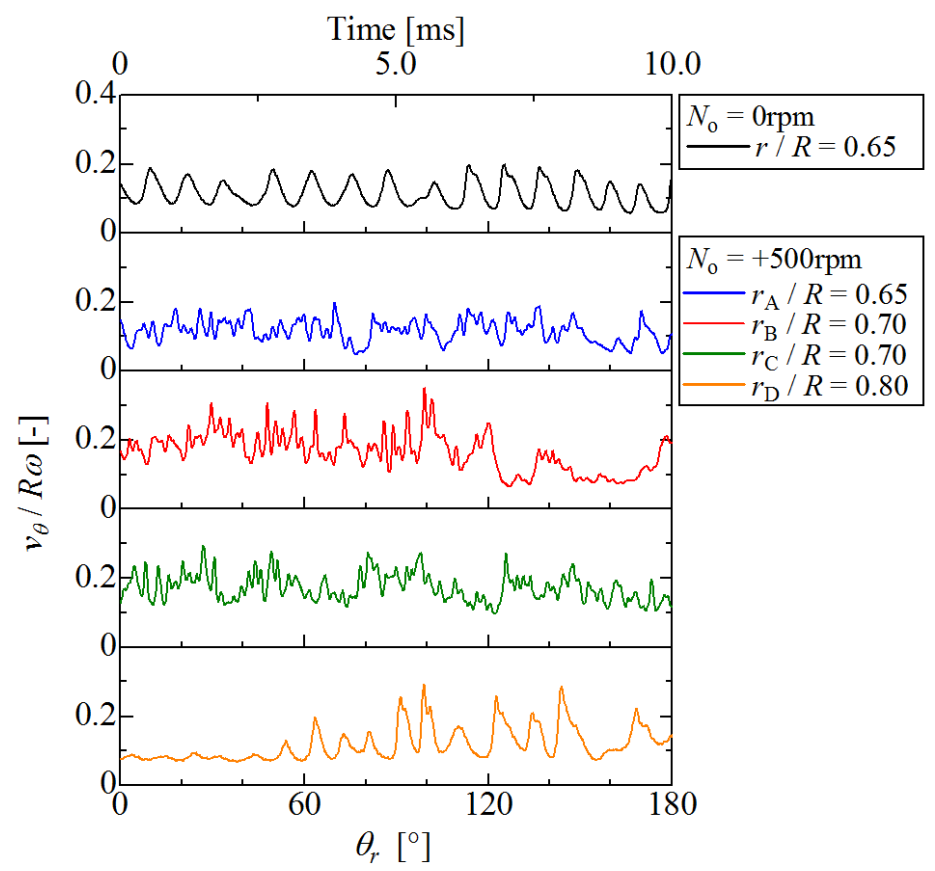

Figure 6. Time histories of $v_{\theta}$ at transient region $(N=+3000 \mathrm{rpm})$.

The low-frequency disturbances are expected to be generated in this region, where the velocity changes most and the outward radial velocity component is maximum at around $\theta=45^{\circ}$ by adding the orbital motion on the rotating disk. Therefore, the origin of the low-frequency disturbances is considered to be associated with the instability of the streamline curvature [9]. The transient vortices in the outer region are destroyed by these low-frequency disturbances, because the periodicity of the fluctuations in velocity associated with the transient vortices diminishes in the outer region. The periodic fluctuations due to transient vortices are broken as shown in the time histories in Figure 6 except at the OD section $\left(r_{\mathrm{D}} / R=0.80\right)$. A detailed investigation will be conducted on this aspect in future work. Also, as the orbital speed increases, the fluctuation intensity in the turbulence region becomes larger except at the OA section. 
In Figure 4(b), for opposite orbital and rotational directions, the onset in the increase in the intensity of fluctuations moves with the shift in the boundary layer transition point as for coincident directions. The low-frequency disturbances in the central region of the disk are not observed in any section and the boundary layer flow in the central region remains laminar state for any negative orbital velocity. As the orbital speed increases, the fluctuating intensity in the turbulence region becomes larger except at the OB section.

\section{Conclusions}

The effects of orbital motion on the velocity field of boundary layer flow over a rotating disk were investigated experimentally. The velocity field at a fixed orbital angular section measured by a hot-wire anemometer show shifts at the transition points and changes in velocity gradients in consequence of the orbital motion. We conclude that the structure of the 3-dimensional boundary layer flow is deformed elliptically and displaced in a certain direction that is not in the orbital radial direction. This displacement depends on the direction of rotation.

For coincide orbital and rotational directions, there are regions where the intensity of fluctuations in velocity increases rapidly, departing from the mean velocity profile in normally laminar regions. These disturbances are characterized by low frequency and high amplitude. The transient vortices, which form streaks on the coating of films, are considered to be destroyed by low-frequency disturbances. However, for opposite orbital and rotational directions, the low-frequency disturbances are not observed at any section.

Concerning the effects occurring in the turbulence region, the fluctuating intensity becomes larger as the orbital speed increases except in a certain region. These are observed irrespective of the direction of rotation. However, the expected location of the region also depends on the direction of rotation.

\section{Acknowledgements}

This study is supported by Tokyo Electron Kyushu Inc. Useful discussions with Mr. Kurishima, H. and Mr. Kudo, K. at Tokyo Electron Kyushu Inc. are appreciated.

\section{References}

[1] Schlichting, H. (1955) Boundary Layer Theory. MCGRAW-HILL, New York, 193199.

[2] Reed, H.L. and Saric, W.S. (1989) Stability of Three Dimensional Boundary Layers. Annual Review of Fluid Mechanics, 21, 235-284. https://doi.org/10.1146/annurev.fl.21.010189.001315

[3] Gregory, N.J., Stuart, T. and Walker, W.S. (1955) On the Stability of Three-Dimensional Boundary Layers with Application to the Flow Due to a Rotating Disk. Philosophical Transactions of the Royal Society of London, A248, 155-199. https://doi.org/10.1098/rsta.1955.0013

[4] Kohama, Y. (1984) Study on Boundary Layer Transition of a Rotating Disk. Acta Mechanica, 50, 193-199. https://doi.org/10.1007/BF01170959 
[5] Imayama, S., Alfredsson, P.H. and Lingwood, R.J. (2014) On the Laminar Turbulent Transition of the Rotating-Disk Flow: the Role of Absolute Instability. Journal of Fluid Mechanics, 745, 132-163. https://doi.org/10.1017/jfm.2014.80

[6] Munekata, M., Jobi, N., Ikebe, K. and Yoshikawa, H. (2012) Effects of Orbital Motion on the Boundary Layer Flow on a Spinning Disk. Open Journal of Fluid Dynamics, 2, 187-194. https://doi.org/10.4236/ojfd.2012.24A020

[7] Munekata, M., Jobi N., Kubo, K. and Yoshikawa, H. (2013) Characteristics of Transient Vortices in the Boundary Layer on a Rotating Disk under Orbital Motion. J. Thermal Science, 22, 600-605. https://doi.org/10.1007/s11630-013-0668-0

[8] Munekata, M., Kubo K. and Yoshikawa, H. (2015) Visualization of Traveling Vortices in the Boundary Layer on a Rotating Disk under Orbital Motion. Open Journal of Fluid Dynamics, 5, 17-25. https://doi.org/10.4236/ojfd.2015.51003

[9] Itoh, N. (1998) Theoretical Description of Instability Waves in Flow on a Rotating Disk. Transactions of the Japan Society for Aeronautical and Space Sciences, 40, 262-279.

\section{Submit or recommend next manuscript to SCIRP and we will provide best} service for you:

Accepting pre-submission inquiries through Email, Facebook, LinkedIn, Twitter, etc. A wide selection of journals (inclusive of 9 subjects, more than 200 journals)

Providing 24-hour high-quality service

User-friendly online submission system

Fair and swift peer-review system

Efficient typesetting and proofreading procedure

Display of the result of downloads and visits, as well as the number of cited articles

Maximum dissemination of your research work

Submit your manuscript at: http://papersubmission.scirp.org/

Or contact ojfd@scirp.org 